Teologia i Moralność, volumen 14(2019), numer 1(25)

doi: $10.14746 /$ tim.2019.25.1.12

ORCID: 0000-0003-2846-0992

ZBIGNIEW FORMELLA

Università Pontificia Salesiana, Roma

\title{
Intervento psico-educativo alla luce della teoria bio-ecologica di Urie Bronfenbrenner
}

\section{Introduzione}

Nel contributo intendo presentare il concetto di intervento psico-educativo nella dimensione ecologica applicata al processo di crescita della persona, con particolare riferimento all'età dello sviluppo scolastico (dai 6 ai 18 anni). In questa tappa della vita, significativa sia dal punto di vista individuale che sociale, l'interazione dell'essere umano con l'ambiente che lo circonda non può essere inquadrata in schemi deterministici del tipo stimolo-risposta, tipici del paradigma biologico, e del puro volontarismo e/o liberalismo purtroppo sempre più presenti nelle proposte educative della società odierna. Voglio offrire una lettura psico-pedagogica in una prospettiva sia psicologica che pedagogica con particolare riferimento alla teoria ecologica di U. Bronfenbrenner, che ritengo molto utile nella visione integrata dello sviluppo e dell'educazione della persona in divenire.

\section{Cambiamenti socio-educative nell'epoca odierna}

Indipendentemente delle proprie credenze e/o convinzioni è necessario notare certi cambiamenti che stanno avvenendo, nelle diverse dimensioni, all'interno della nostra società. Analizzando la letteratura che tratta questi argomenti (Albiero 2011; Aboujaoude 2011; Galimberti 2013; 2017; Sartori 2015; Cassese 2018) e mettendo in risalto il mio personale punto di vista, mi permetto di raggruppare i cambiamenti, che ritengo più importanti e significativi per la nostra vita, in alcune affermazioni-chiave: 
- Il concetto di famiglia non è più uguale per tutti - quest'affermazione è ormai diventata un dato di fatto sia a livello concettuale (legislativo, in alcuni paesi) che a livello sociale (usanze e atteggiamenti quotidiani). L'aspetto che preoccupa maggiormente sono le conseguenze psico-educative a breve termine e quelle sociali a lungo termine per le nuove generazioni. Finché si tratta di eventi statisticamente insignificanti, possiamo concordare che al livello comunitario il problema rimane marginale. Quando, invece, sia i numeri che le convinzioni personali cominciano ad essere significative per l'opinione pubblica - diventa legittimo porsi la domanda - ma la famiglia e il suo ruolo in che direzione si sposta? Certamente rimane valida l'attenzione al discorso promosso dai protagonisti di queste proposte - in altre parole, diventa importante chiedersi - chi promuove certi concetti e perché? Quando il discorso riguarda le fragilità umane, spesso legate alle esperienze negative vissute, sia la discussione che le risposte possono essere influenzate da opinioni ideologiche, politiche, economiche, ecc. Rimane comunque attuale la domanda: i cambiamenti all'interno della famiglia che ripercussioni avranno per il futuro delle nuove generazioni e per la futura società umana? (Gambini 2011; Recalcati 2014).

- Il concetto di relazione interpersonale non è più uguale per tutti. Sia i cambiamenti all'interno delle società che la diffusione nella quotidiana presenza della dimensione virtuale hanno influenzato fortemente le quantità e le qualità delle relazioni tra le persone in tutte le aree della vita. Un esempio eclatante potrebbe essere il cambiamento che sta avvenendo del concetto dell'amicizia. Si è passati, infatti, da una visione "storica" dell'amicizia, cioè quella tradizionale, in un certo senso esclusiva perché provata nel tempo, ad un semplice click online che dichiara di "essere il tuo amico". Sarebbe interessante studiare come questa nuova modalità di dichiarare amicizia influisce sul concetto stesso dell'amicizia che sta cambiando in modo particolare tra le giovane generazioni (Gardner, Davis 2014; Crepet 2016; Riva 2016).

- I concetti di studio, di apprendimento e di insegnamento stanno mutando notevolmente e non sono più legati ad un'unica modalità condivisa nel mondo scolastico ed accademico. Nei tempi di Internet sia l'apprendimento che l'insegnamento, la ricerca scientifica e la modalità di intervento, hanno aperto nuove opportunità, sia nel senso dello sviluppo che delle criticità. Il concetto di studio, di apprendimento e di insegnamento stanno cambiando in modo significativo (Cangià 2014; Roncaglia 2018). Lo sviluppo delle possibilità di studiare, di laurearsi e di lavorare attraverso il mondo virtuale cambiano non solo le modalità di poter raggiungere gli scopi personali, ma cambiano pian paino la dimensione 
umana dal punto di vista cognitivo, affettivo e scoiale (Simone 2012; Tegmark 2018).

- Nel campo educativo si sta perdendo un necessario equilibrio tra la dimensione emancipante e quella socializzante. Questo disequilibrio lo avvertiamo sia nelle proposte concettuali che operative riguardanti il campo dell'apprendimento e dell'insegnamento. Si potrebbe definire un vero e proprio disagio nel saper distinguere la vera scienza dall'ideologia applicata al raggiungimento dei propri scopi. Sembra che quest'ultima cominci a dominare la prima (Brancaccio, Bracci 2019). Sarebbe interessante scoprire il vero ruolo delle cosiddette "lobby" impegnate nel promuovere diverse ricerche con lo scopo non sempre e/o non solo scientifico. Inoltre, diventa sempre più difficile stabilire il confine tra il servizio della scienza per lo sviluppo della società umana. Da una parte la globalizzazione delle diverse problematiche (specialmente quelle di carattere sociale) potrebbe aiutare ad affrontarle per il bene di tanti, dall'altra parte gli interessi dei singoli potenti e delle aziende multinazionali tendono ad influenzare sempre di più sia il contenuto che le forme dell'emancipazione e socializzazione. Se è vero che nel passato la scelta della direzione più emancipante che socializzante (o viceversa) nell'educazione era dettata dalle forze politiche, ideologiche che economiche, oggi domina "l'individuazione manipolata", cioè i pochi (potenti), grazie ai mezzi a disposizione illudono di poter essere se stessi nella propria autorealizzazione. Le conseguenze di questa certa "utopia manipolata" è il crescente "narcisismo personalizzato" e "consumismo affettivo" nelle scelte sia individuali che comunitarie delle persone, sempre più osservate nella società globale (Ritzer 2017; Frezza 2017).

\section{Il contributo della visione bio-psico-ecologica di Urie Bronfenbrenner}

Urie Bronfenbrenner ${ }^{1}$ nella sua visione psico-sociale dello sviluppo umano ha introdotto tre cambiamenti: l'importanza della teoria, l'interazione persona-

\footnotetext{
${ }^{1}$ Urie Bronfenbrenner (1917-2005) - psicologo statunitense. Studiò dapprima psicologia e musica alla Cornell University e in seguito si laureò in psicologia dello sviluppo mentale alla Harvard University e infine nel 1942 ottenne il Ph.D. alla University of Michigan. Dopo appena 24 ore dal dottorato si arruolò nelle forze armate americane impegnate nella seconda guerra mondiale, durante la quale adempì al servizio di psicologo. Una volta terminata la guerra iniziò la carriera di docente universitario, dapprima come assistente di psicologia e poi come professore alla Cornell University nella Facoltà di Sviluppo umano, studi familiari e psicologia. Creatore del modello ecologico con il quale intende l'ambiente di sviluppo del bambino come una serie di cerchi concentrici, legati tra loro da relazioni.
} 
contesto e il legame tra la scienza e la politica. Di seguito mi soffermerò, in modo particolare, sul secondo aspetto.

Le prime esposizioni sistematiche del modello di Bronfenbrenner risalgono al 1970 e furono successivamente modificate con due grandi riformulazioni integrative pubblicate nell'Handbook of Child Psychology del 1983 e del 1998 (Bronfenbrenner, Evans 2000, p. 116).

Bronfenbrenner pone al centro del suo studio la comprensione profonda di ciò che succede alla persona che si trova a vivere e crescere all'interno di un ambiente:

l'ecologia dello sviluppo umano implica lo studio scientifico del progressivo adattamento reciproco tra un essere umano attivo che sta crescendo e le proprietà, mutevoli, delle situazioni ambientali immediate in cui l'individuo in via di sviluppo vive, anche nel senso di definire come questo processo è determinato dalle relazioni esistenti tra le varie situazioni ambientali e dai contesti più ampi di cui le prime fanno parte (Bronfenbrenner 1979, p. 21).

Questa definizione presa dalla maggiore monografia del nostro Autore sottolinea tre punti principali del suo concetto. Prima di tutto, la persona che sta crescendo viene considerata come un "essere umano attivo" cioè un'entità dinamica, capace di riflessione e di intenzionalità. Non è una realtà chiusa o immobile, ancora meno una tabula rasa che viene plasmata dal suo ambiente circostante. Questa visione è rispettosa dell'individuo qualunque sia la sua età. Il secondo punto è il progressivo adattamento reciproco tra la persona e l'ambiente che si realizza in un'interazione bidirezionale. La relazione tra la persona e l'ambiente è caratterizzata dalla reciprocità. Il terzo punto amplifica il concetto di ambiente che non si riduce all'unico contesto in cui la persona si trova a vivere fisicamente come si è soliti fare nella psicologia dello sviluppo. L'ambiente ecologico è costituito da una serie di strutture concentriche incluse l'una nell'altra come le bambole russe. Esso va dall'ambiente circostante a quello più lontano.

Lo sviluppo, essendo il risultato dell'azione reciproca della persona e dell'ambiente, invece di prediligere l'una o l'altra parte, si focalizza sull'identificazione delle dinamiche interattive ossia $\mathrm{i}$ "processi interattivi tra determinate qualità della persona e specifiche caratteristiche dell'ambiente» (Bronfenbrenner 2005, p. 96). Il nostro Autore ne presenta particolarmente due tipi: i processi di interazione sociale e le attività complesse. Il primo caso si riferisce alle interazioni che avvengono tra la persona in via di sviluppo e le altre persone, generalmente di età maggiore, talvolta con i pari, ma raramente più giovani. Il secondo caso riguarda il coinvolgimento in attività e compiti progressivamente più complessi che costituisce il motore principale dello svi- 
luppo. Questi due tipi di processi permettono a Bronfenbrenner di sottolineare l'importanza di due componenti che determinano la qualità dell'ambiente: le persone presenti da una parte e dall'atra le caratteristiche fisiche e simboliche.

Questo pensiero è stato applicato in diversi modi alla realtà della famiglia e al mondo del lavoro che ci coinvolgono tutti. L'Autore parte da un'osservazione legata alla specificità del genere umano: "i contesti della famiglia e del lavoro rappresentano delle attività centrali per la sopravvivenza dell'uomo [...]. Sono soprattutto i genitori che ci insegnano per primi in che modo gli ambienti si possano trasformare e così facendo ci preparano al mondo del lavoro. Senza una presenza dei genitori estesa nel tempo saremmo del tutto incapaci di vivere e agire nell'ambiente che abbiamo creato" (Bronfenbrenner 2005, p. 261).

Per rendere adeguatamente il proprio servizio, i genitori hanno bisogno del sostegno della scuola, dei datori di lavoro, dei legislatori e dell'intera società. Infatti, quello che fanno è per la sopravvivenza della nostra specie e per il suo futuro. "Se non riconosciamo il ruolo chiave della genitorialità per la crescita dei figli nella nostra società e se non diamo tempo ai genitori per svolgere il loro compito, dovremo presto affrontare la disgregazione del nostro stesso tessuto sociale" (Bronfenbrenner 2005, p. 267). Descrivendo la realtà statunitense, Bronfenbrenner osserva che "il disordine nella vita dei nostri bambini, giovani e famiglie pervade ormai troppi ambiti fondamentali della nostra esistenza: le nostre case, il sistema sanitario, l'organizzazione dei servizi per l'infanzia, il gruppo dei pari, le scuole, il quartiere in cui viviamo, il posto di lavoro, i mezzi di trasporto e comunicazione" (Bronfenbrenner 2005, p. 195). Personalmente mi sembrano molto attuali le sue osservazioni anche per la società odierna, in modo particolare nella sua dimensione sociale ed educativa.

Il concetto di ecologia è stato usato per la prima volta da Barker e Wright (1954) nell'ambito dello sviluppo umano ma loro non hanno spiegato come cambia l'ambiente e come questo cambiamento influisce sullo sviluppo della persona. Così, per rispondere alle ricerche sullo sviluppo senza contesto, Bronfenbrenner propose il modello che chiama ecologia dello sviluppo umano che sottolinea l'adattamento reciproco tra l'essere in crescita e il suo ambiente immediato con i contesti più ampi che lo includono (Bronfenbrenner 1977b, p. 514). Lo sviluppo umano così inteso si realizza nell'ambiente ecologico la cui struttura richiama una serie di bambole russe. «L'ambiente ecologico è concepito topologico, e cioè come una serie ordinata di strutture concentriche incluse l'una nell'altra» (Bronfenbrenner 1977b, p. 514; 1979b, p. 22).

Visto che la definizione dell' ecologia dello sviluppo umano pone al centro l'interazione tra la persona in crescita, gli individui con chi ha delle relazioni faccia-a-faccia da una parte e l'ambiente nel quale si trovano dall'altra, le caratteristiche di un paradigma ecologico includono le due parti. Per la persona, 
le qualità personali e gli attributi capaci di strutturare l'ambiente vengono definiti caratteristiche rilevanti per lo sviluppo (Bronfenbrenner 1992b, p. 218$-225)$.

Successivamente, Bronfenbrenner ha collocato al centro del suo modello i processi prossimali e, a partire dal 1993 e dal 1998, ha proposto il modello denominato: Processo-Persona-Contesto-Tempo, (P-P-C-T) come il disegno di ricerca appropriato per la sua teoria che da ecologica diventa bioecologica.

L'obiettivo principale perseguito dall'Autore era di indicare come le caratteristiche individuali e il contesto spazio-temporale influenzano lo sviluppo attraverso i processi prossimali.

Come fece il nostro Autore nella prima fase definendo cosa si intende per "ecologia dello sviluppo" (Bronfenbrenner 1979b), in questa ultima fase, definì il "modello bioecologico" come un sistema teorico in continua evoluzione adatto per lo studio scientifico dello sviluppo umano nel tempo (Bronfenbrenner, Morris 2006, p. 793; Bronfenbrenner 2001b). Si vede qui la traiettoria del modello che si chiarisce e cresce come un organismo vivente. Nella prima fase, si insisteva sull'importanza del contesto nello sviluppo umano e poi si è passati all'individuazione delle caratteristiche bio-psicofisiologiche rilevanti per lo sviluppo nella seconda fase. Nell'ultima fase, il modello include la dimensione temporale legata alla persona in crescita.

Due proprietà interdipendenti definiscono fondamentalmente il modello bioecologico.

La prima:

Particolarmente nelle sue fasi iniziali, ma anche nel corso dell'esistenza, lo sviluppo dell'uomo si realizza attraverso interazioni reciproche, progressivamente più complesse, che si verificano tra un organismo umano, in attiva e continua evoluzione, e le persone, i simboli e gli oggetti che fanno parte dell'ambiente più prossimo. Per poter risultare efficace, quest'interazione deve avvenire in modo sufficientemente regolare e per un periodo di tempo esteso. Tale tipo di interazione costante, che ha luogo nell'ambiente immediato, viene chiamata processo prossimale" (Bronfenbrenner, Morris 1998, p. 996).

La seconda:

La forma, l'intensità, il contenuto e la direzione di un processo prossimale che produce sviluppo varia sistematicamente come funzione congiunta:

- delle caratteristiche della persona in via di sviluppo (comprese le sue eredità genetiche);

- dell'ambiente - sia immediato sia più remoto - nel quale si realizzano i processi evolutivi;

- della natura dei risultati dello sviluppo che si vuole considerare; 
- degli elementi di continuità e di trasformazione che avvengono nell'ambiente nel corso del tempo, durante la vita della persona e in base al periodo storico nel quale la persona si trova a vivere (Bronfenbrenner, Morris 1998, p. 996).

La prima proposizione dà un'ampia definizione del processo prossimale e la seconda precisa le dinamiche e i soggetti che intervengono nello sviluppo vanno lette insieme. Lo sviluppo umano viene dunque generato da un insieme di forze di origine personale ed ambientale che agiscono concordemente nel tempo in un circolo di relazioni nell'ambiente circostante. Il processo prossimale funziona dunque come il motore dello sviluppo umano (Bronfenbrenner, Evans 2000, p. 118).

La prospettiva bioecologica applicata alle interazioni ci porta a prendere in considerazione l'alunno, i suoi compagni, l'insegnante, gli educatori e gli altri adulti significativi presenti nell'ambiente circostante. Le capacità cognitive, le caratteristiche socio-emozionali e motivazionali e la visione della persona come agente attivo in grado di contribuire al suo stesso sviluppo possono guidare la nostra lettura dell'intervento psico-educativo in questo campo (Bronfenbrenner 1992b, p. 202-203).

\section{L'intervento bassato sulle potenzialità personali e ambientali}

Nella costruzione dell'intervento psico-educativo rivolto alla persona in età di sviluppo scolastico viene sempre consigliato, a partire dalle potenzialità sia della persona stessa che dell'ambiente circostante, che si promuova lo sviluppo di "quello che c'è" per poter "acquistare quello che non c'è".

La società si aspetta dall'educazione lo svolgimento di alcune funzioni, tra le quali l'emancipazione e la socializzazione. La funzione socializzante si lega alla preparazione delle persone nell'assumere diversi ruoli nella e per la società, in modo da non alterarne l'equilibrio. In altri termini, si può parlare di una funzione adattiva. In questo caso, l'agire educativo o formativo dovrebbe trasmettere un sistema di valori e un atteggiamento in modo da dotare le persone di conoscenze e abilità socialmente utili, tenendo presenti i vari compiti e ruoli richiesti dalla società. La funzione emancipante e liberatrice è legata all'organizzazione delle condizioni nell'ambiente in cui vive la persona in sviluppo affinché il suo potenziale possa liberarsi e realizzarsi. Vista sotto quest'ottica l'educazione dovrebbe favorire lo sviluppo della persona, prendersi cura delle sue individualità, delle differenze, e far crescere le sue competenze specifiche. Entrambe le funzioni sono in continuo "conflitto costruttivo" tra un educatore maturo, capace di accompagnamento adeguato nei confronti di un ragazzo, bisognoso di essere accompagnato (Formella 2015). 
La meta finale di ogni intervento psico-educativo di carattere trasversale è la modificazione dello stile di vita piuttosto che l'eliminazione del problema. Avvicinarsi ad un ragazzo che presenta un problema comportamentale o nell'apprendimento cercando di comprenderne la finalità, significa offrire la possibilità di esprimersi ed essere ascoltati. Questo processo rappresenta, di conseguenza, la possibilità di interagire con gli altri, compiere scelte e partecipare attivamente alla vita quotidiana.

L'educatore e/o insegnante in questo caso, viene chiamato a saper percepire, trattare ed accompagnare con competenza il percorso della crescita. Nella società odierna essere un educatore efficace non è certamente facile (Formella 2009). Forse non lo era neanche prima ma rimane valido sottolineare l'importanza della maturità operativa di un adulto che lavora con i giovani indicando e condividendo la proposta fatta da G. Salonia (2008) dei tre importanti "segnali stradali" per chi fa o farà questo mestiere di guida degli altri:

a) l'orizzonte dell'interiorità creativa,

b) l'orizzonte della condivisione,

c) l'orizzonte della responsabilità.

Nel linguaggio scout, indicare l'orizzonte vuol dire, proporre la direzione, ma senza i particolari. Esprimo l'augurio che questi indicatori possano essere validi sia per la formazione di chi si occupa di progettare un intervento psicoeducativo che per una visione trasversale della relazione educativa, nel processo di accompagnamento nella crescita del ragazzo, definito nel linguaggio tradizionale "educazione".

Alla fine, ritengo opportuno ricordare il pensiero del prof. Pio Scilligo, apprezzato docente di diverse generazioni di psicologi, psicoterapeuti ed educatori dell'Ateneo Pontificio Salesiano e non solo, secondo il quale:

la persona umana è intelligente e sociale. Data la sua intelligenza, il modo di rispondere a qualsiasi evento è determinato in gran parte dai significati che ad essi l'individuo attribuisce, dalle scelte possibili che intravede e dalla previsione degli effetti derivanti sia dagli eventi che dall'azione. Il quanto è sociale, i suoi pensieri e le sue azioni in modo implicito ed esplicito si attuano nel contesto di altre persone. Perciò, i processi mentali di fondo riguardanti la personalità sono strettamente collegati con il conoscere sociale in tutto gli ambienti: nella rappresentazione mentale di sé, degli altri e della situazione nella quale si attuano i processi interpersonali; nei processi adottati nel costruire e ricostruire le impressioni e quello che si sperimenta; nel formulare valutazioni e attribuzioni ed altri giudizi riguardanti persone ed eventi nel mondo sociale; infine nell'effetto del conoscere sociale sulla condotta sociale (Scilligo 2005, p. 41-42). 


\section{Conclusione}

Troppo spesso nell'operare sociale, educativo che psicologico, chi agisce si concentra nel voler risolvere il problema offrendo soluzioni "pronte", intervenendo secondo moduli prestabiliti e volendo subitamente diminuire i danni. Questi tentativi sono marcati dal "peccato originale" della nostra società moderna, cioè "adesso e subito (veloce)". Abbiamo difficolta a rispettare i tempi, prendendo in considerazione il saggio concetto del Qoèlet - "c'è un tempo per ogni cosa" (Qo 3,1-2). Da una parte è vero che l'educazione, la crescita, lo sviluppo del ragazzo avviene tramite la relazione con la persona adulta significativa, dall'altra è anche vero che l'educazione è una questione di rispetto per il tempo della maturazione. Nel mio discorso ho aggiunto anche la terza dimensione, cioè l'ottimismo, che vuol dire il basarsi sulle potenzialità presenti e quelle possibili da acquisire sia per l'azione educativa (in altre parole l'intervento personale ed ambientale) che per il processo di crescita in vista di un protagonismo di questo processo da parte dell'educando.

\section{PSYCHOEDUCATIONAL INTERVENTION IN THE LIGHT OF THE BIO- ECOLOGICAL THEORY OF URIE BRONFENBRENNER}

\section{SUMMARY}

This article presents the interpretation of the bioecological model of the American psychologist Urie Bronfenbrenner, applied to the growth process, especially during the school period. It should be noted that any psychoeducational intervention is designed to change both in the person and in the surrounding environment with which the person is in constant exchange. An important role in the process of change is played by a significant adult figure, which is often a parent, teacher, educator. Given that this process is taking place in modern society, which is subject to constant changes, it seems important to recall some basic dynamics that lead to healthy individual and social growth.

Keywords: change; ecology; Urie Bronfenbrenner; psychoeducational intervention

Słowa kluczowe: zmiana; ekologia; Urie Bronfenbrenner; interwencja psychoedukacyjna 


\section{Bibliografia}

Aboujaoude E. (2011), Virtually You: The Dangerous Powers of the E-Personality, New York-London.

Albiero P. a cura di (2011), Il benessere psicosociale in adolescenza. Prospettive multidisciplinary, Roma.

Barker R.G., Wright H.F. (1954), Midwest and its Children. The Psychological Ecology of an American Town, Evanston [USA, Illinois].

Brancaccio E., Bracci G. (2019), Il discorso del potere. Il premio Nobel per l'economia tra scienza, ideologia e politica, Milano.

Bronfenbrenner U. (1977b), Toward an Experimental Ecology of Human Development, "American Psychologist" 32(7), 513-531.

Bronfenbrenner U. (1979), The Ecology of Human Development. Experiments by Nature and design, Cambridge, [USA, Massachusetts] (Trad. it.), Ecologia dello sviluppo umano, Bologna (2002).

Bronfenbrenner U. (1992b). Ecological systems theory, in: Six theories of child development: Revised formulations and current issues, ed. R. Vasta, London, p. 187-249.

Bronfenbrenner U. (2001b). The bioecological theory of human development, in: International encyclopedia of the social and behavioral sciences, Eds. N.J. Smelser, P.B. Baltes, Vol. 10, Oxford [UK], pp. 6963-6970.

Bronfenbrenner U. (2005), Making Human Beings Human. Bioecological Perspectives on Human Development, Thousand Oaks.

Bronfenbrenner U., Morris P.A. (1998), The ecology of developmental processes, in: Handbook of Child Psychology: Vol. 1. Theoretical models of human development, $5^{\text {th }}$ Ed., Eds. W. Damon, R.M. Lerner, New York, p. 993-1028.

Bronfenbrenner U., Evans G.W. (2000), Developmental Science in the 21 ${ }^{\text {st }}$ Century: Emerging Questions, Theoretical Models, Research Designs and Empirical Findings, "Social Development" 9(1), p. 115-125.

Bronfenbrenner U., Morris P.A. (2006), The bioecological model of human development, in: Handbook of child psychology, Vol. 1: Theoretical models of human development $6^{\text {th }}$ ed., Eds. W. Damon, R.M. Lerner, New York, p. 793-828.

Cangià C. (2014), Generazione tech. Crescere con i nuovi media, Firenze.

Cassese S. (2018), La democrazia e i suoi limiti, Milano.

Crepet P. (2016), Baciami senza rete. Buone ragioni per sottrarsi alla seduzione digitale, Milano.

Formella Z. (2009), L'educatore maturo nella comunicazione efficace, Roma.

Formella Z. (2015), Psicologia dell'intervento educativo, Roma.

Frezza E. (2017), MalaScuola. «Gender», affettività, emozioni. Il sistema «educativo» per abolire la ragione e manipolare $i$ nostri figli, Roma.

Galimberti U. (2013), I miti del nostro tempo, Milano.

Galimberti U. (2017), Il tramonto dell'Occidente nella lettura di Heidegger e Jaspers, Milano.

Gambini P. (2011), Adolescenti e famiglia affettiva. Percorsi d'emancipazione, Milano.

Gardner H., Davis K. (2014), Generazione App. La testa dei giovani e il nuovo mondo digitale, Milano.

Recalcati M. (2014), Il complesso di Telemaco. Genitori e figli dopo il tramonto del padre, Milano.

Riva G. (2016), Selfie. Narcisismo e identità, Bologna.

Salonia G. (2008), Maturità, in: Dizionario di Scienze dell'Educazione, coord. J.M. Prellezzo, C. Nanni, G. Malizia, Roma, p. 714-717.

Sartori G. (2015), La corsa verso il nulla. Dieci lezioni sulla nostra società in pericolo, Milano.

Sciligo P. (2005), La nuova sinfonia dei molti Sé, Roma.

Simone R. (2012), Presi nella rete. La mente ai tempi del web, Milano. 
Ritzer G. (2017), La McDonaldizzazione della produzione, Roma.

Roncaglia G. (2018), L'età della frammentazione. Cultura del libro e scuola digitale, Bari.

Tegmark M. (2018), Vita 3.0. Essere umani nell'era dell'intelligenza artificiale, Milano.

Prof. dr Zbigniew Formella - salesiano, dottorato in scienze umanistiche, professore ordinario all'Università Pontificia Salesiana a Roma, direttore della cattedra di Psicologia dell'Educazione. Le sue ricerche scientifiche riguardano le problematiche giovanili di carattere psico-educativo. Possiede diverse pubblicazioni in merito, sia libri che articoli. E-mail: formella@unisal.it 